\title{
Software to Compute Infinitesimal Symmetries of Exterior Differenial Systems, with Applications
}

\author{
PAUL H. M. KERSTEN \\ Faculty of Applied Mathematics, University of Twente, P.O. Box 217, 7500 AE Enschede, \\ The Netherlands
}

(Received: 26 July 1988)

\begin{abstract}
A description is given of a software package to compute symmetries of partial differential equations, using computer algebra. As an application, the computation of higher-order symmetries of the classical Boussinesq equation is given leading to the recursion operator for symmetries in a straightforward way. Nonlocal symmetries for the Federbush model are obtained yielding the linearization of the model.
\end{abstract}

AMS subject classifications (1980). 53B50, 35Q20, 68B99.

Key words. Computer algebra, symmetries, recursion operators.

\section{Introduction and General}

The huge amount of essential standard computations necessary to obtain the Lie algebra of infinitesimal symmetries of a differential equation or an exterior differential system, leads in a natural way to the question: how to do these computations with the help of a computer?

We shall describe some software to carry through these computations on a computer system. We use REDUCE [1], a symbolic language based on LISP [2].

In Section 2, we shall indicate what kind of problems one comes across mathematically, and we shall mention the procedures which are constructed to execute these computations on a computer system.

The reader is assumed not to have knowledge of REDUCE or LISP in order to understand Section 1.

In Section 3, we shall give a general description of the procedures, suited for a user of the developed software. For more detailed information, the reader is referred to [3].

Schwartz [4] constructed a software package quite similar to ours, but based on doing computations completely automatic. However, it is our experience that due to this, in some sense, over-automation, the system is very time-consuming and leads to expression-swell.

In Section 4 we give an application of the developed software in the con- 
struction of higher-order symmetries and the recursion operator of the classical Boussinesq equation.

Finally, in Section 5, we deal with nonlocal symmetries and the linearization of the Federbush model.

A differential equation is described as a subset $Y \subset J^{k}(M, N)$ being the zero set of

$$
\begin{aligned}
& F: J^{k}(M, N) \rightarrow \mathbb{R}^{c} \quad(c \in \mathbb{N}), \\
& \zeta \in Y \rightarrow 0,
\end{aligned}
$$

where $J^{k}(M, N)$ is the $k$ th order jet bundle of $M$ and $N . M$ is the manifold of independent variables with local coordinate presentation $\left(x^{a}\right)=\left(x^{1}, \ldots, x^{m}\right)$ and $N$ is the manifold of dependent variables with local coordinate representation $\left(z^{\mu}\right)=\left(z^{1}, \ldots, z^{n}\right)$, while a local coordinate system on $J^{k}(M, N)$ is given by

$$
\left(x^{a}, z^{\mu}, z_{a}^{\mu}, \ldots, z_{a_{1}, \ldots, a_{k}}^{\mu}\right),
$$

with $a, a_{1}, \ldots$ running from 1 to $m, \mu$ running from 1 to $n$.

The total partial derivative vector fields on $J^{k}(M, N)$ are defined by

$$
D_{a}^{(k)}=\partial_{a}+z_{a}^{\mu} \partial_{z^{\mu}}+\cdots+z_{a a_{1}, \ldots, a_{k-1}}^{\mu} \partial_{z_{a_{1}, \ldots, a_{k-1}}}
$$

The $r$ th prolongation $Y^{r}$ of a differential equation is defined as the zero set of

$$
p^{r} F: J^{k+r}(M, N) \rightarrow \mathbf{R}^{q},
$$

where $p^{\prime} F$ is defined by

$$
\zeta \in J^{k+r}(M, N) \rightarrow\left(F^{h}(\zeta),\left(D_{a}^{k+r} F^{h}\right)(\zeta), \ldots,\left(D_{a_{1}, \ldots, a_{r}}^{k+r} F^{h}\right)(\zeta)\right) .
$$

In $(1.4 \mathrm{a}, \mathrm{b}) D_{a}^{k+r} F^{h}$ denotes the Lie derivative of the function $F^{h}$ with respect to the total partial derivative vector field $D_{a}^{k+r} ;\left(D_{a}^{(k+r)} F^{h}\right), \ldots,\left(D_{a_{1}, \ldots, a_{r}}^{(k+r)} F^{h}\right)$ are the differential consequences of $F^{h}(h=1, \ldots, c)$.

We use the notation

$$
D_{a_{1}, \ldots, a_{r}}^{(k+r)}=D_{a_{1}}^{(k+r)} \ldots D_{a_{r}}^{(k+r)} .
$$

In terms of differential forms, a differential equation of order $k$ can be described by a closed ideal $I$ of differential forms defined on $J^{k}(M, N)$, where closed means $d(I) \subset I$.

Without loss of generality, we can assume that a closed ideal $I$ is generated by homogeneous forms $\alpha_{1}, \ldots, \alpha_{m}$ and their exterior derivatives $\mathrm{d} \alpha_{1}, \ldots, \mathrm{d} \alpha_{m}$. sometimes we call $I$ an exterior differential system. In most problems, we construct the ideal $I$ of differential forms associated with a differential equation in the following way:

start at the basis contact one-forms and solve each of the

$$
F^{h}(\zeta)=0 \quad(h=1, \ldots, c)
$$


for some $z_{a_{1} \ldots, a_{k}}^{\mu}$ and substitute; the closed ideal $I$ is then generated by these new one-forms and their exterior derivatives.

In effect, we construct the restriction of the contact one-forms to the differential equation.

The $r$ th prolongation of the closed ideal $I$, denoted by $D^{\prime} I$ is defined as the closed ideal of differential forms of $J^{k+r}(M, N)$, generated by

$$
\begin{aligned}
& \alpha_{1}, \ldots, \alpha_{m}, \mathrm{~d} \alpha_{1}, \ldots, \mathrm{d} \alpha_{m} \\
& D_{a}^{(k+r)} \alpha_{1}, \ldots, D_{a}^{(k+r)}\left(\mathrm{d} \alpha_{m}\right), \ldots, D_{a_{1}, \ldots, a_{r}}^{(k+r)} \alpha_{1}, \ldots, D_{a_{1}, \ldots, a_{r}}^{(k+r)}\left(\mathrm{d} \alpha_{m}\right) .
\end{aligned}
$$

An infinitesimal symmetry (for short: symmetry) of an exterior differential system $I$ is a vector field $V$ which satisfies the condition

$$
L_{V} I \subset I
$$

where $L_{V}$ denotes the Lie derivate with respect to the vector field $V$. Condition (1.7) is called the symmetry condition and the procedure INFSYM of Section 2 reflects this.

In order to describe higher-order (infinitesimal) symmetries, we have to pass to the infinite jet bundle $J(M, N)$ of $M$ and $N$, where the local coordinates are given by

$$
x^{a}, z^{\mu}, z_{a}^{\mu}, \ldots, z_{a_{1}, \ldots, a_{r}}^{\mu}, \ldots
$$

A smooth function [5] $f$ is a function defined on $J(M, N)$ such that $f$ factors through $J^{k}(M, N)$ for some $k$, meaning that $f$ is dependent only on a finite number of variables, denoted by $f \in C^{\infty}(J(M, N))$. A smooth vector field on $J(M, N)$ has a coordinate presentation

$$
X=\xi^{a} \partial_{a}+\eta^{\mu} \partial_{z^{\mu}}+\cdots+\eta_{a_{1}, \ldots, a_{k}}^{\mu} \partial_{z_{a_{1}, \ldots, a_{k}}^{\mu}}+\cdots
$$

where

$$
\xi^{a}, \eta^{\mu}, \ldots, \eta_{a_{1}}^{\mu}, \ldots, a_{k}, \ldots, \in C^{\infty}(J(M, N)) .
$$

Since every smooth function $f$ factors through some $J^{k}(M, N)$, there are no problems concerning convergence in taking the Lie derivative

$$
X f=L_{X} f
$$

of a smooth function $f$ with respect to a vector field $X$ defined on $J(M, N)$.

Total partial derivative vector fields on $J(M, N)$ are given in natural local coordinates by

$$
\mathrm{D}_{a}=\partial_{a}+z_{a}^{\mu} \partial_{z^{\mu}}+\cdots+z_{a a_{1}, \ldots, a_{k}}^{\mu} \partial_{z_{a}^{\mu}, \ldots a_{k}}+\cdots
$$

If the exterior differential system $I$ is generated by the forms

$$
\alpha_{1}, \ldots, \alpha_{m}, \mathrm{~d} \alpha_{1}, \ldots, \mathrm{d} \alpha_{m},
$$


then the infinitely prolonged exterior differential system $D^{\infty} I$ is generated by

$$
\begin{aligned}
& \alpha_{1}, \ldots, \alpha_{m}, \mathrm{~d} \alpha_{1}, \ldots, \mathrm{d} \alpha_{m} \\
& D_{a} \alpha_{1}, \ldots, D_{a} \mathrm{~d} \alpha_{m} \\
& \vdots \\
& D_{a_{1}, \ldots, a_{k}} \alpha_{1}, \ldots, D_{a_{1}, \ldots, a_{k}} \mathrm{~d} \alpha_{m} \\
& \vdots
\end{aligned}
$$

where in (1.13)

$$
D_{a_{1}, \ldots, a_{k}}=D_{a_{1}} D_{a_{2}}, \ldots, D_{a_{k}} .
$$

Now, a Lie-Bäcklund transformation or generalized symmetry is a vector field $V$ defined on $J(M, N)$ such that

$$
L_{V}\left(D^{\infty} I\right) \subset D^{\infty} I .
$$

Due to the construction of $D^{\infty} I(1.13)$, the total derivative vector fields $D_{a}(1.11)$ satisfy (1.14) in an obvious way. In general, the vector field $T$ defined by

$$
T=\xi^{a} D_{a}
$$

where $\xi^{a} \in C^{\infty}(J(M, N))$, satisfies (1.14).

Two vector fields $X_{1}, X_{2}$ defined on $J(M, N)$ are equivalent [7] if there are smooth functions $\xi^{a} \in c^{\infty}(J(M, N))$ such that

$$
X_{1}=X_{2}+\xi^{a} D_{a}
$$

Combination of (1.15) and (1.16) shows that we can restrict the study of Lie-Bäcklund transformations to vertical vector fields $V$, i.e., those vector fields for which

$$
\left(\pi_{M}\right) * V=0,
$$

where in (1.17) $\pi_{M}$ is the natural projection of $J(M, N)$ on $M$.

The Lie-Bäcklund transformations which are vertical vector fields, can be shown to have the following coordinate representation [5]

$$
V=\eta^{\mu} \partial_{z^{\mu}}+\eta_{a}^{\mu} \partial_{z_{a}^{\mu}}+\cdots+\eta_{a_{1}, \ldots, a_{k}}^{\mu} \partial_{z_{a_{1}}^{\mu}, \ldots, a_{k}}+\cdots,
$$

where the functions $\eta_{a}^{\mu}, \ldots, \eta_{a_{1}, \ldots, a_{k}}^{\mu}, \ldots \in C^{\infty}(J(M, N))$ are defined by

$$
\begin{aligned}
& \eta_{a}^{\mu}=D_{a} \eta^{\mu} \\
& \vdots \\
& \eta_{a_{1}, \ldots, a_{k}}^{\mu}=D_{a_{1}, \ldots, a_{k}} \eta^{\mu} \\
& \vdots
\end{aligned}
$$

The functions $\eta^{\mu}(1.18)$ are called the generating functions of the vector field $V$ and we shall use the notation 


$$
V=\eta^{\mu} \partial_{z^{\mu}}+\cdots
$$

instead of (1.18) and (1.19).

The vector field $V(1.18),(1.19)$ commutes with the total derivative vector fields $D_{a}$ (1.11).

From these observations, we conclude that it suffices to satisfy

$$
L_{V} I \subset D^{\infty} I
$$

instead of (1.14).

Finally, since the vector field $V(1.20)$ is generated by smooth functions $\eta^{\mu}$, i.e., dependent on a finite number of variables, condition, $(1.21)$ reduces to the condition

$$
L_{V} I \subset D^{\prime} I \text { for some } r \text {. }
$$

Condition (1.22) will be used in the construction of the Lie-Bäcklund transformations in Section 4.

\section{Basic Ideas}

To compute infinitesimal symmetries we start with a closed ideal $I$ of differential forms, defined on an $n$-dimensional space $\mathbf{R}^{n}=\{(x(1), \ldots, x(n))\}$ and generated by homogeneous differential forms $\alpha(i)(i=1, \ldots, m)$,

$$
I=\langle\alpha(1), \ldots, \alpha(m)\rangle .
$$

The vector field $V$, defined by

$$
V=\sum_{i=1}^{n} F(i) \partial_{x(i)},
$$

where $F(i)(i=1, \ldots, n)$ are functions defined on $\mathbf{R}^{n}$, is an infinitesimal symmetry of (2.1) if $V$ satisfies the condition (1.7)

$$
L_{V} I \subset I .
$$

Equation (2.3) is equivalent to

$$
L_{v} \alpha(k)+\sum_{j=1}^{m} \gamma(k, j) \alpha(j)=0 \quad(k=1, \ldots, m),
$$

where $\gamma(k, j)$ are suitable differential forms.

Elimination of the coefficients of $\gamma(k, j)(k, j=1, \ldots, m)$ from these conditions (2.3) results in an overdetermined, linear and homogeneous system of partial differential equations for the functions $F(i)(i=1, \ldots, n)$, the components of the vector field $V$. The procedure INFSYM generates this overdetermined system of partial differential equations.

The nature of these overdetermined systems obtained from condition (2.3) is, 
in many practical situations, very specific $[8,9,10]$, and one comes across very special types of partial differential equations which have to be solved.

The left-hand sides of these partial differential equations are stored in REDUCE as values of an operator VER, so the values VER(1), .., VER(TOTAL) are just these left-hand sides. A solution of (2.3) is a set of $n$ functions $F(i) \quad(i=1, \ldots, n)$ such that the evaluations of $\operatorname{VER}(1), \ldots, \operatorname{VER}(T O T A L)$ become zero.

The functions $F(i)$ are given in REDUCE without explicit occurrence of their variables. They are stored as values in an association list DEPL!*.

By $F\left(^{*}\right)$ we denote some function: $F$ (index).

By $D F\left(F\left({ }^{*}\right),{ }^{*}\right)$ we denote some derivative of a function $F($ index) with respect to a list of variables: $D F(F$ (index), list of variables).

We assume the partial differential equations to be of the following structure

function: $F\left(^{*}\right)$

derivative: $D F\left(F\left({ }^{*}\right),{ }^{*}\right)$

coefficient: an algebraic expression not containing a function $F\left(^{*}\right)$ or a $D F\left(F\left({ }^{*}\right),{ }^{*}\right)$

term: coefficient ${ }^{*}$ function / coefficient ${ }^{*}$ derivative

equation: term / term + equation.

We shall now describe the most frequently occurring cases, which arise in practical situations. The procedure FINES searches for such situations and constructs the general solution to such equations. The procedures ONETERMSOL, SPLIT, described in [11] are just special situations, dealt with by the procedure FINES.

In examples, we shall use a REDUCE-like notation; by $\operatorname{VER}(\cdot)$ we mean one of the $\operatorname{VER}(1), \ldots, \operatorname{VER}(T O T A L)$.

\section{CASE A}

A partial differential equation is of the polynomial type in one (or more) of the variables, the functions $F\left(^{*}\right)$ appearing in this equation are independent of this variable. As a consequence, each of the coefficients of the polynomial has to be zero, and the partial differential equation decomposes into some new and smaller equations.

EXAMPLE 2.1. The partial differential equation is

$$
\operatorname{VER}(\cdot):=X(1)^{* *} 2^{*} D F(F(1), X(2))+X(1)^{*} F(2)
$$

where in (2.4) $F(1), F(2)$ are functions independent of $X(1)$.

As a consequence, the coefficients of the polynomial in $X(1)$ have to be zero, i.e., $D F(F(1), X(2))$ and $F(2)$.

So $(2.4)$ is equivalent to 
$\operatorname{VER}(\cdot):=D F(F(1), X(2))$

$\operatorname{VER}(\cdot):=F(2)$.

CASE B

The partial differential equation $\operatorname{VER}(\cdot)$ represents some derivative of a function $F($ ).

In general

$$
\operatorname{VER}(\cdot):=D F\left(F\left(^{*}\right), X\left(i_{1}\right), k_{1}, \ldots, X\left(i_{r}\right), k_{r}\right),
$$

a mixed $\left(k_{1}+\cdots+k_{r}\right)$ th order derivative, while the $k_{i}$ 's are not present in the case $k_{i}=1$.

The general solution of $(2.6)$ is

$$
F\left({ }^{*}\right)=\sum_{s=1}^{r} \sum_{t=0}^{k_{s}-1} F\left(i_{s, t}\right)^{*} X\left(i_{s}\right)^{t}
$$

whereas in (2.7) $F\left(i_{s, t}\right)$ depends on the same variables as $F()$, except $X\left(i_{s}\right)$ $\left(t=0, \ldots, k_{s}-1, s=1, \ldots, r\right)$.

EXAMPLE 2.2.

$$
\operatorname{VER}(\cdot):=D F(F(1), X(1), X(2)) \text {. }
$$

The general solution to this equation is given by

$$
F(1):=F(2)+F(3)
$$

where $F(2)$ depends on the same variables as $F(1)$, except $X(1)$, while $F(3)$ depends on the same variables as $F(1)$, except $X(2)$.

\section{CASE C}

The partial differential equation $\operatorname{VER}(\cdot)$ contains a function $F()$ depending on all variables present as arguments of some other function(s) $F\left({ }^{*}\right)$, occurring in this equation, whereas there is no derivative of the function $F()$ present in the equation. The partial differential equation can be solved for this function $F()$.

EXAMPLE 2.3. The equation is

$$
\operatorname{VER}(\cdot):=X(1)^{*} F(1)+X(2)^{*} D F(F(2), X(1))
$$

where in (2.10), $F(1), F(2)$ are dependent on $X(1), X(2), X(3)$.

The solution is

$$
F(1):=\left(-X(2)^{*} D F(F(2), X(1))\right) / X(1),
$$




\section{CASE D}

In the partial differential equation, there is a derivative of a function $F()$ with respect to variables which are not present as argument of any other function $F\left({ }^{*}\right)$, while the coefficient of $F()$ is a number. By the assumption that $X(1), \ldots, X(n)$ appear as polynomials, the partial differential equation can be integrated.

EXAMPLE 2.4. Let the partial differential equation be given by

$$
\operatorname{VER}(\cdot):=D F(F(1), X(3))+X(2)^{*} F(2)
$$

where

$F(1)$ depends on $X(1), X(2), X(3)$,

$F(2)$ depends on $X(1), X(2)$.

The solution to $(2.12)$ is

$$
F(1):=-X(2)^{*} X(3)^{*} F(2)+F(3)
$$

whereas $F(3)$ depends on $X(1), X(2)$ and is independent of $X(3)$.

\section{CASE E}

In the partial differential equation a specific variable $X(i)$ is present just once as an argument of some function $F\left(^{*}\right)$. By appropriate differentiation, one might arrive at a simple equation, which can be solved. Evaluation of the original equation can result in an equation which can be solved.

\section{EXAMPLE 2.5 .}

$$
\operatorname{VER}(\cdot):=X(2)^{*} D F(F(1), X(2), X(3))+X(3)^{*} F(2)
$$

where

$F(1)$ depends on $X(1), X(2), X(3)$,

$F(2)$ depends on $X(1), X(2)$.

Differentiation, with respect to $X(3)$ twice results in

$$
\operatorname{VER}(\cdot):=X(2)^{*} D F(F(1), X(2), X(3), 3)
$$

The solution to (2.16) is (CASE B)

$$
F(1):=F(3)^{*} X(3)^{* *} 2+F(4)^{*} X(3)+F(5)+F(6) .
$$


where

$F(3), F(4), F(5)$ are dependent on $X(1), X(2)$,

$F(6)$ depends on $X(1), X(3)$.

Evaluation of (2.15) leads to

$$
\begin{aligned}
\operatorname{VER}(\cdot):= & 2^{*} X(2)^{*} X(3)^{*} D F(F(3), X(2))+ \\
& +X(2)^{*} D F\left(F(4), X(2)+X(3)^{*} F(2) .\right.
\end{aligned}
$$

Due to CASE A, FINES constructs two new equations

$$
\begin{aligned}
& \operatorname{VER}(\cdot):=2^{*} X(2)^{*} D F(F(3), X(2))+F(2), \\
& \operatorname{VER}(\cdot):=X(2)^{*} D F(F(4), X(2)) .
\end{aligned}
$$

The effect of FINES applied to (2.15) will be (2.17), (2.19).

The procedure FINES is then again useful to handle Equations $(2.19 \mathrm{a}, \mathrm{b})$. This last step is not executed automatically.

In many practical problems, one is able to solve the overdetermined system of partial differential equations (2.3) using the methods described in CASE A, B, C, $\mathrm{D}, \mathrm{E}$, and some additional considerations due to the nature of the specific problem at hand.

The final result is a set of $n$ expressions for the functions $F(1), \ldots, F(n)$, the components of the vector field $V(2.2)$.

In these expressions there are several integration constants $F\left({ }^{*}\right)$ due to CASES B, D, E and even sometimes free functions [11] or functions which have to satisfy a genuine differential equation [12]. From the representation of this general Vector Field $V$ the procedure VFGEN constructs the set of GENerators of the Lie algebra of infinitesimal symmetries.

\section{General Description of the Procedures}

A general description of the procedures, used in the construction of the Lie algebra of infinitesimal symmetries of exterior differential systems is given. The source code of the procedures is given in [3]. A description of LIEDF, INFSYM, GEFORM, FINES, FSOLV, ELIM, VFGEN is given.

In the construction of the overdetermined system of partial differential equations, which has to be satisfied by the components of the vector field $V$ in order to be an infinitesimal symmetry, we use the package constructed by Gragert [13]. This package is used to carry through computations of differential geometric nature.

We list below some global data required by this package.

D!@DIF: the dimension of the vector space

OPERATOR VNAT: the coordinates of the vector space 
OPERATOR UIT: to describe basis forms, e.g., $\mathrm{d} x \equiv \mathrm{UIT}(1)$. (UIT product is the Dutch word for wedgeproduct)

The procedures described in [13] and called within the procedure bodies of this section, are EXDF, IP, NORMDIF, MULFORM, !@BEVATOP, OPCOEFF, OPL.

Moreover, throughout this section we assume the following global data to exist

DEPL!*, FPKTEL, TOTAL, LIOV

OPERATOR VER; OPERATOR F.

TOTAL: the number of partial differential equations describing the conditions on the components of the vector field $V$.

FPKTEL: the total number of functions introduced in solving the overdetermined system.

DEPL!*: an association list available in REDUCE to store the arguments of functions.

LIOV: the name of the key to an element of the association list DEPL!* where all the independent variables of the overdetermined system are stored.

VER,F: the partial differential equations for the functions $F(I) \quad(I=$ $1, \ldots, \mathrm{D}$ !@DIF i.e., the components of the vector field $V$ are stored as values of the operator VER: VER(1),.., VER(TOTAL).

EXAMPLE 3.1. The value of DEPL!* is

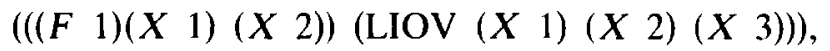

if there is only one function $F(1)$ dependent on $X(1), X(2)$, while there are $X(1)$, $X(2), X(3)$ as variables in the system.

$\operatorname{LIEDF}\left({ }^{*} 1,{ }^{*}\right)$

The procedure computes the Lie derivative of a differential form with respect to a vector field.

parameters:

${ }^{*} 1$ : the vector field,

$*_{2}$ : the differential form.

The procedure body is just the definition of the Lie derivative

$$
L_{v} \alpha=V \mathrm{~d} \alpha+\mathrm{d}(V \alpha) .
$$

$\operatorname{INFSYM}\left(* 1,{ }^{*} 2,{ }^{*} 3\right)$

The procedure constructs the overdetermined system of partial differential equations for the components of the vector field $V$. 


\section{parameters:}

${ }^{*} 1$ : the number of the first differential form $\operatorname{ALFA}\left({ }^{*} 1\right)$ on which condition (2.3) is applied.

*2: the number of the last differential form ALFA $(* 2)$ on which condition (2.3) is applied.

Condition (2.3) is applied to $\operatorname{ALFA}\left({ }^{*} 1\right), \ldots, \operatorname{ALFA}\left({ }^{*} 2\right)$

*3: the total number of differential forms $\operatorname{ALFA}(1), \ldots, \operatorname{ALFA}\left({ }^{*} 3\right)$ generating the exterior differential system.

global data:

OPERATOR ALFA, $\operatorname{ALFA}(1), \ldots, \operatorname{ALFA}\left({ }^{*} 3\right)$, $F(1), \ldots, F(D ! @ D I F)$.

procedure calls:

NORMDIF, MULFORM, OPCOEFF, !@BEVATOP, OPL, CLEARKVALUE, LIEDF, GEFORM.

result:

The result is the number of partial differential equations constructed by the procedure.

effect:

The overdetermined system of partial differential equations associated to the condition

$$
L_{V} \alpha(i) \in I, \quad\left(i={ }^{*} 1, \ldots,{ }^{*} 2\right)
$$

are stored as values of $\operatorname{VER}(1), \ldots, \operatorname{VER}(T O T A L)$.

side effects:

$1^{\circ}$ Message: THERE EXIST N EQUATIONS FOR ALFA (M)

$2^{\circ}$ In case there are denominators introduced in the construction of the overdetermined system, there will be a message:

CREATION OF DENOMINATOR.

$\operatorname{GEFORM}\left({ }^{*} 1,{ }^{*} 2\right)$

The procedure constructs a GEneral differential FORM of order less than 4, with unspecified coefficients.

parameters:

* 1 : an index to distinguish general differential forms

${ }^{*} 2$ : the order of the differential form $\left({ }^{*} 3\right)$. 
global data:

OPERATOR CO: used to denote the unspecified coefficients of the differential form.

result:

The result is the required differential form of order $* 2$.

$\operatorname{FINES}\left({ }^{*} 1\right)$

The construction of the general solution of an equation $\operatorname{VER}\left({ }^{*} 1\right)$ or construction of an equivalent system.

parameter:

*1: the number of the partial differential equation, $\operatorname{VER}\left({ }^{*} 1\right)$ to which the procedure is applied.

global data:

FIDEPT,

ARRAY FIPKA (20),

ARRAY FIPKA1 (the number of variables in the system).

FIDEPT: a global whose value avoids a recursion of the procedure FINES of depth greater than 2 .

FIPKA(20): an array to construct the coefficients of a polynomial (highest power $\leqslant 20$ ).

FIPKA1(. .): an array whose elements contain information about the number of occurrences of a variable as argument of functions $F\left({ }^{*}\right)$ in $\operatorname{VER}(* 1)$.

Note: the dimension 20 for the array FIPKA, turned out to be suitable for practical purposes. A user can redefine this array himself taking a dimension $>20$.

procedure calls:

OPCOEFF, STRPOLY, FSOLV, CLEARKVALUE.

effects:

The construction of the general solution to the partial differential equation, or the construction of an equivalent system.

side effect:

there will be messages about actions and searches of the procedure:

CASE A: VER(*1) BREAKS INTO VER( $(\cdot), \ldots, \operatorname{VER}(\cdot)$ BY:

(...). 
CASE B: HOMOGENEOUS INTEGRATION OF: $\left(D F\left(F^{*}\right),{ }^{*}\right)$

CASE C VER( $\left.{ }^{*} 1\right)$ IS SOLVED FOR : $\left(F^{*}\right)$

CASE D: INHOMOGENEOUS INTEGRATION OF: $\left(D F\left(F^{*}\right),{ }^{*}\right)$

CASE E: SEARCH FOR A DIFFERENTIATION

( )

TOTAL :=

\section{ELIMINATION OF VARIABLE(S) :FINES}

If the search in CASE D (Section 1) is not successful there will be one of the following messages:

$1^{\circ}$ : MORE THAN ONE MAXIMAL DF(F $\left.\left({ }^{*}\right),{ }^{*}\right)$, in case there are two or more derivatives having all variables present in the equation as their argument.

$2^{\circ}$ : COEFFICIENT OF THE DERIVATIVE IS NOT A NUMBER.

In these situations no further action is taken.

Note: a $\operatorname{DF}\left(F\left({ }^{*}\right),{ }^{*}\right)$ is called MAXIMAL in case $F\left(^{*}\right)$ depends on all the variables present in the partial differential equation $\operatorname{VER}(* 1)$

$\operatorname{FSOLV}\left({ }^{*} 1,{ }^{*}\right)$

the procedure solves an equation $\operatorname{VER}\left({ }^{*} 1\right)$ for a function $F\left({ }^{*} 2\right)$.

parameters:

${ }^{*} 1$ : The number of the partial differential equations $\operatorname{VER}\left({ }^{*} 1\right)$.

*2: The number of the function $F\left({ }^{*} 2\right)$.

procedure call:

OPL

result:

NIL

effect:

The solution of the partial differential equation.

$\operatorname{ELIM}\left({ }^{*} 1,{ }^{*}\right)$

The purpose of the procedure is to replace the sum of two functions which occur in the system; they often arise from integration constants. 
parameters:

*1: the index of the function $F\left({ }^{*} 1\right)$ which has to be eliminated

*2: the index of the other function.

result:

NIL

effect: if allowed then $F\left(^{*} 1\right)$ is replaced by

$-F\left({ }^{*} 2\right)+F\left(^{* *}\right)\left(F\left(^{* *}\right)\right.$ : a new function)

side effect:

in case the elimination is not allowed a message:

WRONG ELIMINATION.

In this situation the elimination is not carried through.

VFGEN $\left({ }^{*} 1\right)$

The procedure decomposes the general solution of the overdetermined system into the generating vector fields of the Lie algebra of infinitesimal symmetries.

parameters:

*1: The dimension of the vector fields to be constructed.

global data:

VFPKTEL: $\quad$ a counter for vector fields

VFPKTRL: $\quad$ a list of new variables

OPERATOR D the components of the vector fields

OPERATOR VF: the name of the Vector Fields

OPERATOR VFV: the name of an auxiliary vector field.

\section{Higher Order Symmetries and the Recursion Operator of the Classical Boussinesq Equation}

As a first application of the developed software, higher-order symmetries are computed for the classical Boussinesq equation and from one of these higherorder symmetries the recursion operator is constructed in a straightforward way. The same result was obtained by Itô [14] in a nonconstructive way.

The classical Boussinesq equation is written as the following system of partial differential equations

$$
\begin{aligned}
& u_{t}=\partial_{x}\left(u v+\sigma v_{x x}\right) \quad(\sigma \text { parameter }), \\
& v_{t}=\partial_{x}\left(u+\frac{1}{2} v^{2}\right)
\end{aligned}
$$

First of all we note that Equations (4.1) are graded in the following way 


$$
\begin{aligned}
& \operatorname{deg}(u)=1 ; \quad \operatorname{deg}(v)=\frac{1}{2}, \\
& \operatorname{deg}(x)=-\operatorname{deg}\left(\partial_{x}\right)=-\frac{1}{2}, \\
& \operatorname{deg}(t)=-\operatorname{deg}\left(\partial_{t}\right)=-1
\end{aligned}
$$

and partial derivatives $u_{x}, u_{1}, \ldots$ are graded accordingly; for instance $\operatorname{deg}\left(v_{x x x}\right)=2$.

In order to construct higher-order symmetries of (4.1), we introduce the closed ideal $I$ of differential forms on

$$
\begin{aligned}
\mathbb{R}^{1.3} & =\{(x(1), \ldots, x(13))\} \\
& =\left\{\left(x, t, u, v, v_{01}, v_{1}, v_{01}, v_{2}, v_{11}, v_{02}, v_{21}, v_{12}, v_{03}\right)\right\}
\end{aligned}
$$

generated by the 7-one-forms $\alpha_{1}, \ldots, \alpha_{7}$

$$
\begin{aligned}
& \alpha_{1}=\mathrm{d} u-u_{1} \mathrm{~d} x-u_{01} \mathrm{~d} t, \\
& \alpha_{2}=\mathrm{d} v-v_{1} \mathrm{~d} x-v_{01} \mathrm{~d} t, \\
& \alpha_{3}=\mathrm{d} v_{1}-v_{2} \mathrm{~d} x-v_{11} \mathrm{~d} t, \\
& \alpha_{4}=\mathrm{d} v_{01}-v_{11} \mathrm{~d} x-v_{02} \mathrm{~d} t, \\
& \alpha_{5}=\mathrm{d} v_{2}-v_{3} \mathrm{~d} x-v_{21} \mathrm{~d} t, \\
& \alpha_{6}=\mathrm{d} v_{11}-v_{21} \mathrm{~d} x-v_{12} \mathrm{~d} t, \\
& \alpha_{7}=\mathrm{d} v_{02}-v_{12} \mathrm{~d} x-v_{03} \mathrm{~d} t
\end{aligned}
$$

and their exterior derivatives $\mathrm{d} \alpha_{1}, \ldots, \mathrm{d} \alpha_{7}$.

In (4.3), we introduced an abbreviation for the partial derivatives, p.e., $v_{21}=v_{x x t}, u_{1}$ and $v_{3}$ are obtained from Equations (4.1).

We furthermore constructed the third prolongation $D^{3} I$ of the ideal $I(4.3)$, defined on

$\mathbb{R}^{25}=\left\{\left(x, t, u, v, \ldots, \hat{u}_{11}, \hat{u}_{31}, \hat{u}_{12}, \hat{v}_{32}, \hat{v}_{13}, \hat{v}_{33}, \ldots, v_{06}\right)\right\}$ generated by $\alpha_{1}, \ldots, \alpha_{7}$ defined by (4.3)

and

$$
\begin{aligned}
& \alpha_{8}=\mathrm{d} u_{01}-u_{11} \mathrm{~d} x-u_{02} \mathrm{~d} t, \\
& \alpha_{9}=\mathrm{d} v_{21}-v_{31} \mathrm{~d} x-v_{22} \mathrm{~d} t, \\
& \alpha_{10}=\mathrm{d} v_{12}-v_{22} \mathrm{~d} x-v_{13} \mathrm{~d} t, \\
& \alpha_{11}=\mathrm{d} v_{03}-v_{13} \mathrm{~d} x-v_{04} \mathrm{~d} t, \\
& \alpha_{12}=\mathrm{d} u_{02}-u_{12} \mathrm{~d} x-u_{03} \mathrm{~d} t, \\
& \alpha_{13}=\mathrm{d} v_{22}-v_{32} \mathrm{~d} x-v_{23} \mathrm{~d} t, \\
& \alpha_{14}=\mathrm{d} v_{13}-v_{23} \mathrm{~d} x-v_{14} \mathrm{~d} t, \\
& \alpha_{15}=\mathrm{d} v_{04}-v_{14} \mathrm{~d} x-v_{05} \mathrm{~d} t,
\end{aligned}
$$




$$
\begin{aligned}
& \alpha_{16}=\mathrm{d} u_{03}-u_{13} \mathrm{~d} x-u_{04} \mathrm{~d} t, \\
& \alpha_{17}=\mathrm{d} v_{23}-v_{33} \mathrm{~d} x-v_{24} \mathrm{~d} t, \\
& \alpha_{18}=\mathrm{d} v_{14}-v_{24} \mathrm{~d} x-v_{15} \mathrm{~d} t, \\
& \alpha_{19}=\mathrm{d} v_{05}-v_{15} \mathrm{~d} x-v_{06} \mathrm{~d} t,
\end{aligned}
$$

where $u_{11}, v_{31}, u_{12}, v_{32}, u_{13}, v_{33}$ are obtained from (4.1) by total partial differentiation with respect to $t$.

Now let $V$ be the vertical vector field defined on $\mathbb{R}^{25}$ by

$$
V=F(3) \partial_{u}+F(4) \partial_{v}+\cdots,
$$

where in (4.5) the functions $F(3), F(4)$ are supposed to be dependent on $x, t, u, v, \ldots$; up to and including third-order derivatives in $v$, and up to and including second-order derivatives in $u$, i.e.;

$$
x, t, u, v, u_{01}, v_{1}, v_{01}, v_{2}, v_{11}, v_{21}, v_{12}, v_{03}, u_{02}
$$

The (higher-order) symmetry condition on $V$, i.e.,

$$
L_{V}(I) \subset D^{3} I
$$

where $L_{V}$ denotes the Lie derivative with respect to $V$, leads to an overdetermined system of 14 partial differential equations INFSYM $(1,7,19)$.

We solved this overdetermined system by symbolic integration, using the developed software described before. We also took great advantage of the grading (4.2) of the system of partial differential equation (4.1).

The complete solution of (4.7) under condition (4.6) consists of linear combinations of eight vector fields $Y_{1}, \ldots, Y_{8}$.

Before writing down the result, we choose $u, v$ and their $x$-derivatives as independent variables on the submanifold of the infinite jet bundle $J^{\infty}(x, t ; u, v)$ defined by (4.1) and the differential consequences.

In these variables the result is given by

$$
Y_{i}=Y(i, 3) \partial_{u}+Y(i, 4) \partial_{v} \quad(i=1, \ldots, 8),
$$

where

$$
\begin{aligned}
& Y(1,3):=V_{3}{ }^{*} \operatorname{SIG}+U_{1}{ }^{*} V+V_{1}{ }^{*} U \quad(\mathrm{SIG}=\sigma), \\
& Y(1,4):=U_{1}+V_{1}{ }^{*} V, \\
& Y(2,3):=U_{1}, \\
& Y(2,4):=V_{1}, \\
& Y(3,3):=T^{*} U_{1}, \\
& Y(3,4):=T^{*} V_{1}+1, \\
& Y(4,3):=\left(X^{*} U_{1}+2^{*} T^{*} V_{3}{ }^{*} \mathrm{SIG}+2^{*} T^{*} U_{1}{ }^{*} V+2^{*} T^{*} V_{1}{ }^{*} U+2^{*} U\right) / 2, \\
& Y(4,4):=\left(X^{*} V_{1}+2^{*} T^{*} U_{1}+2^{*} T^{*} V_{1}{ }^{*} V+V\right) / 2,
\end{aligned}
$$




$$
\begin{aligned}
& Y(5,3):=\left(2^{*} X^{*} V_{3}{ }^{*} \mathrm{SIG}+2^{*} X^{*} U_{1}{ }^{*} V+2^{*} X^{*} V_{1}{ }^{*} U+4^{*} T^{*} U_{3}{ }^{*} \mathrm{SIG}+\right. \\
& +6^{*} T^{*} V_{3}{ }^{*} \mathrm{SIG}^{*} V+12^{*} T^{*} V_{2}{ }^{*} V_{1}{ }^{*} \mathrm{SIG}+3^{*} T^{*} U_{1}{ }^{*}\left(V^{2}+2^{*} U\right)+ \\
& \left.+6^{*} T^{*} V_{1}^{*} V^{*} U+6^{*} V_{2}^{*} \mathrm{SIG}+4^{*} V^{*} U\right) /\left(4^{*} \mathrm{SIG}\right) \text {, } \\
& Y(5,4):=\left(2^{*} X^{*} U_{1}+2^{*} X^{*} V_{1}{ }^{*} V+4^{*} T^{*} V_{3}{ }^{*} \mathrm{SIG}+6^{*} T^{*} U_{1}{ }^{*} V+\right. \\
& \left.+3^{*} T^{*} V_{1}^{*}\left(V^{2}+2^{*} U\right)+V^{2}+4^{*} U\right) /\left(4^{*} \mathrm{SIG}\right) \text {, } \\
& Y(6,3):=\left(2^{*} V_{5}{ }^{*} \mathrm{SIG}^{2}+4^{*} U_{3}{ }^{*} \mathrm{SIG}^{*} V+V_{3}{ }^{*} \mathrm{SIG}^{*}\left(3^{*} V^{2}+5^{*} U\right)+\right. \\
& +9^{*} U_{2}{ }^{*} V_{1}{ }^{*} \mathrm{SIG}+10^{*} V_{2}{ }^{*} U_{1}^{*} \mathrm{SIG}+12^{*} V_{2}{ }^{*} V_{1}{ }^{*} \mathrm{SIG}^{*} V+ \\
& \left.+U_{1}^{*} V^{*}\left(V^{2}+6^{*} U\right)+3^{*} V_{1}^{3 *} \mathrm{SIG}+3^{*} V_{1}^{*} U^{*}\left(V^{2}+U\right)\right) / \mathrm{SIG} \text {, } \\
& Y(6,4):=\left(2^{*} U_{3}{ }^{*} \mathrm{SIG}+4^{*} V_{3}{ }^{*} \mathrm{SIG}^{*} V+7^{*} V_{2}{ }^{*} V_{1}{ }^{*} \mathrm{SIG}+3^{*} U_{1}{ }^{*}\left(V^{2}+U\right)+\right. \\
& \left.+V_{1}^{*} V^{*}\left(V^{2}+6^{*} U\right)\right) / \mathrm{SIG} \\
& Y(7,3):=\left(16^{*} U_{5}{ }^{*} \mathrm{SIG}^{2}+40^{*} V_{5}{ }^{*} \mathrm{SIG}^{2 *} V+120^{*} V_{4}{ }^{*} V_{1}{ }^{*} \mathrm{SIG}^{2}+40^{*}{U_{3}}^{*}\right. \\
& \operatorname{SIG}^{*}\left(V^{2}+U\right)+200^{*} V_{3}^{*} V_{2}^{*} \mathrm{SIG}^{2}+20^{*} V_{3}^{*} \mathrm{SIG}^{*} V^{*} \\
& \left(V^{2}+5^{*} U\right)+80^{*} U_{2}{ }^{*} U_{1}{ }^{*} \mathrm{SIG}+180^{*} U_{2}{ }^{*} V_{1}{ }^{*} \mathrm{SIG}^{*} V+ \\
& +200^{*} V_{2}^{*} U_{1}^{*} \operatorname{SIG}^{*} V+40^{*} V_{2}^{*} V_{1}{ }^{*} \operatorname{SIG}^{*}\left(3^{*} V^{2}+5^{*} U\right)+ \\
& +150^{*} U_{1}^{*} V_{1}^{2 *} \mathrm{SIG}+5^{*} U_{1}^{*}\left(V^{4}+12^{*} V^{2 *} U+6^{*} U^{2}\right)+ \\
& \left.+60^{*} V_{1}^{3 *} \mathrm{SIG}^{*} V+20^{*} V_{1}^{*} V^{*} U^{*}\left(V^{2}+3^{*} U\right)\right) /\left(16^{*} \mathrm{SIG}\right) \text {, } \\
& Y(7,4):=\left(16^{*} V_{5}{ }^{*} \mathrm{SIG}^{2}+40^{*} U_{3}{ }^{*} \operatorname{SIG}^{*} V+40^{*} V_{3}{ }^{*} \operatorname{SIG}^{*}\left(V^{2}+U\right)+80^{*}\right. \\
& U_{2}^{*} V_{1}^{*} \mathrm{SIG}+80^{*} V_{2}^{*} U_{1}{ }^{*} \mathrm{SIG}+140^{*} V_{2}^{*} V_{1}{ }^{*} \mathrm{SIG}^{*} V+ \\
& +20^{*} U_{1}^{*} V^{*}\left(V^{2}+3^{*} U\right)+30^{*} V_{1}^{3 *} \mathrm{SIG}+ \\
& \left.+5^{*} V_{1}^{*}\left(V+12^{*} V^{2 *} U+6^{*} U^{2}\right)\right) /\left(16^{*} \mathrm{SIG}\right) \text {, } \\
& Y(8,3):=\left(4^{*} U_{3}{ }^{*} \mathrm{SIG}+6^{*} V_{3}{ }^{*} \mathrm{SIG}^{*} V+12^{*} V_{2}{ }^{*} V_{1}{ }^{*} \mathrm{SIG}+\right. \\
& \left.+3^{*} U_{1}^{*}\left(V^{2}+2^{*} U\right)+6^{*} V_{1}^{*} V^{*} U\right) /\left(4^{*} \mathrm{SIG}\right) \text {, } \\
& Y(8,4):=\left(4^{*} V_{3}^{*} \mathrm{SIG}+6^{*} U_{1}^{*} V+3^{*} V_{1}^{*}\left(V^{2}+2^{*} U\right)\right) /\left(4^{*} \mathrm{SIG}\right) \text {. }
\end{aligned}
$$

The Lie bracket of the vertical vector fields $Y_{i}(i=1, \ldots, 8)$ is defined by

$$
\left[Y_{i}, Y_{j}\right]^{l}=\mathbb{L}_{Y_{i}}(Y(j, l))-\mathbb{L}_{Y_{j}}(Y(i, l)) \quad(l=3,4) \text {. }
$$

The commutators of the vector fields are given in Table I.

In Table $I, Y_{9}$ is defined by

$$
\begin{aligned}
Y(9,3):= & \left(80^{*} V_{7}{ }^{*} \mathrm{SIG}^{3}+240^{*} U_{5}{ }^{*} \mathrm{SIG}^{2 *} V+20^{*} V_{5}{ }^{*} \mathrm{SIG}^{2 *}\right. \\
& \left(15^{*} V^{2}+14^{*} U\right)+800^{*} U_{4}{ }^{*} V_{1}{ }^{*} \mathrm{SIG}^{2}+840^{*} V_{4}{ }^{*} U_{1}{ }^{*} \mathrm{SIG}^{2}+ \\
& +1800^{*} V_{4}{ }^{*} V_{1}{ }^{*} \mathrm{SIG}^{2 *} V+1400^{*} U_{3}{ }^{*} V_{2}{ }^{*} \mathrm{SIG}^{2}+200^{*} \\
& U_{3}^{*} \mathrm{SIG}^{*} V^{*}\left(V^{2}+3^{*} U\right)+1400^{*} V_{3}{ }^{*} U_{2}{ }^{*} \mathrm{SIG}^{2}+3000^{*} V_{3}{ }^{*} V_{2}{ }^{*} \\
& \mathrm{SIG}^{2 *} V+2250^{*} V_{3}^{*} V_{1}^{2 *} \mathrm{SIG}^{2}+25^{*} V_{3}{ }^{*} \\
& \mathrm{SIG}^{*}\left(3^{*} V^{4}+30^{*} V^{2 *} U+14^{*} U^{2}\right)+1200^{*} U_{2}{ }^{*}
\end{aligned}
$$


Table I

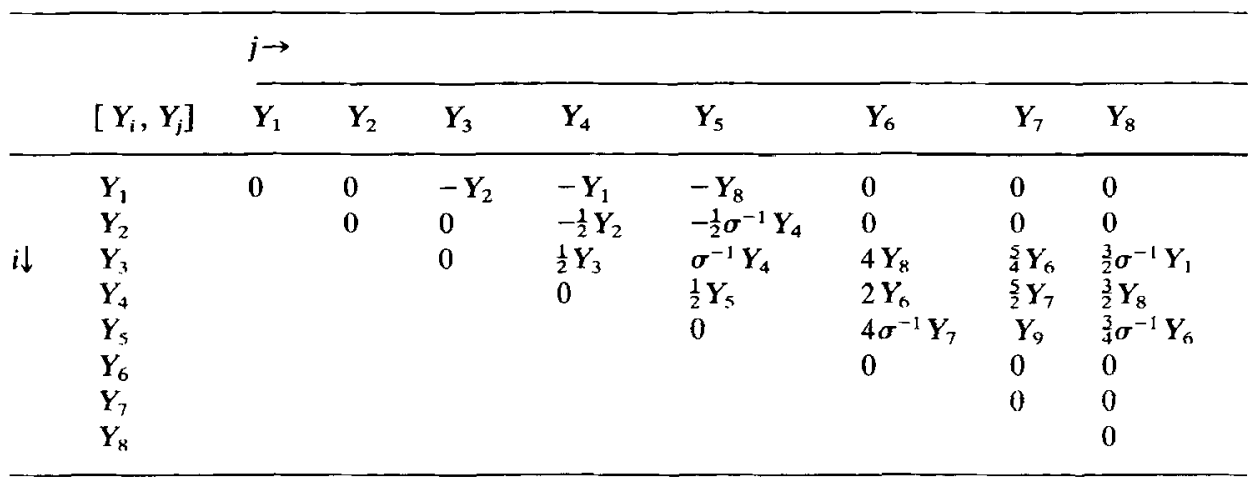

$$
\begin{aligned}
& U_{1}^{*} \mathrm{SIG}^{*} V+50^{*} U_{2}{ }^{*} V_{1}{ }^{*} \mathrm{SIG}^{*}\left(27^{*} V^{2}+26^{*} U\right)+3000^{*} V_{2}^{2 *} V_{1}{ }^{*} \\
& \mathrm{SIG}^{2}+100^{*} V_{2}{ }^{*} U_{1}{ }^{*} \mathrm{SIG}^{*}\left(15^{*} V^{2}+14^{*} U\right)+600^{*} V_{2}{ }^{*} V_{1}{ }^{*} \mathrm{SIG}^{*} V \\
& { }^{*}\left(V^{2}+5^{*} U\right)+1000^{*} U_{1}^{2 *} V_{1}{ }^{*} \mathrm{SIG}+2250^{*} \mathrm{U}_{1}{ }^{*} \mathrm{~V}_{1}^{2 *} \mathrm{SIG}^{*} V+15^{*} \\
& U_{1}{ }^{*} V^{*}\left(V^{4}+20^{*} V^{2 *} U+30^{*} U^{2}\right)+150^{*} V_{1}^{3} \\
& \left.{ }^{*} \mathrm{SIG}^{*}\left(3^{*} V^{2}+5^{*} U\right)+75^{*} V_{1}^{*} U^{*}\left(V^{4}+6^{*} V^{2 *} U_{2}+2^{*} U^{2}\right)\right) / \\
& \left(32^{*} \mathrm{SIG}^{2}\right),
\end{aligned}
$$

$$
\begin{aligned}
& Y(9,4):=\left(80^{*} U_{5}{ }^{*} \mathrm{SIG}^{2}+240^{*} V_{5}{ }^{*} \mathrm{SIG}^{2 *} V+640^{*} V_{4}^{*} V_{1}^{*} \mathrm{SIG}^{2}+100^{*} U_{3}{ }^{*}\right. \\
& \mathrm{SIG}^{*}\left(3^{*} V^{2}+2^{*} U\right)+1000^{*} V_{3}^{*} V_{2}^{*} \mathrm{SIG}^{2}+200^{*} V_{3}^{*} \mathrm{SIG}^{*} V^{*} \\
& \left(V^{2}+3^{*} U\right)+400^{*} U_{2}{ }^{*} U_{1}^{*} \mathrm{SIG}+1200^{*} U_{2}{ }^{*} V_{1}{ }^{*} \mathrm{SIG}^{*} V+ \\
& +1200^{*} V_{2}^{*} U_{1}^{*} \operatorname{SIG}^{*} V+50^{*} V_{2}^{*} V_{1}^{*} \operatorname{SIG}^{*}\left(21^{*} V^{2}+22^{*} U\right)+ \\
& +850^{*} U_{1}^{*} V_{1}^{2 *} \mathrm{SIG}+75^{*} U_{1}^{*}\left(V^{4}+6^{*} V^{2 *} U+2^{*} U^{2}\right)+450^{*} V_{1}^{3 *} \\
& \left.\mathrm{SIG}^{*} V+15^{*} V_{1}^{*} V^{*}\left(V^{4}+20^{*} V^{2 *} U+30^{*} U^{2}\right)\right) /\left(32^{*} \mathrm{SIG}^{2}\right)
\end{aligned}
$$

In order to transform the Lie algebra we introduce

$$
\begin{array}{lll}
Z_{1}=\sigma Y_{5} & W_{1}=Y_{2} & W_{4}=\frac{3}{8} \sigma Y_{6} \\
Z_{0}=Y_{4} & W_{2}=\frac{1}{2} Y_{1} & W_{5}=\frac{3}{2} \sigma Y_{7} \\
Z_{-1}=Y_{3} & W_{3}=\frac{\sigma}{2} Y_{8} & W_{6}=\frac{3}{2} \sigma^{2} Y_{9},
\end{array}
$$

which results in the Lie commutator table given as Table II.

From Table II, we expect that the vector field $Z_{1}$ will give rise to the recursion operator for symmetries.

Equations (4.1) can be written as a Hamiltonian system, i.e.,

$$
\partial_{t}\left(\begin{array}{l}
u \\
v
\end{array}\right)=\left(\begin{array}{cc}
0 & D_{x} \\
D_{x} & 0
\end{array}\right)_{\delta_{v}}^{\delta_{u}}\left(\frac{1}{2} u^{2}+\frac{1}{2} u v^{2}-\frac{1}{2} \sigma v_{x}^{2}\right)
$$

where 
Table II.

\begin{tabular}{lllllllll}
\hline$\left.{ }^{*},{ }^{*}\right]$ & $Z_{1}$ & $Z_{0}$ & $Z_{-1}$ & $W_{1}$ & $W_{2}$ & $W_{3}$ & $W_{4}$ & $W_{5}$ \\
\hline$Z_{1}$ & 0 & $-\frac{1}{2} Z_{1}$ & $-Z_{0}$ & $W_{2}$ & $W_{3}$ & $W_{4}$ & $W_{5}$ & $W_{6}$ \\
$Z_{11}$ & & 0 & $\frac{1}{2} Z_{-1}$ & $\frac{1}{2} W_{1}$ & $W_{2}$ & $\frac{3}{2} W_{3}$ & $2 W_{4}$ & $\frac{5}{2} W_{5}$ \\
$Z_{-1}$ & & & 0 & 0 & $\frac{1}{2} W_{1}$ & $\frac{3}{2} W_{2}$ & $3 W_{3}$ & $5 W_{4}$ \\
$W_{1}$ & & & & 0 & 0 & 0 & 0 & 0 \\
$W_{2}$ & & & & & 0 & 0 & 0 & 0 \\
$W_{3}$ & & & & & & 0 & 0 & 0 \\
$W_{4}$ & & & & & & & 0 & 0 \\
$W_{5}$ & & & & & & & & 0 \\
\hline
\end{tabular}

$$
\Omega^{-1}=\left(\begin{array}{cc}
0 & D_{x} \\
D_{x} & 0
\end{array}\right)
$$

is the sympletic operator and

$$
H=\int_{-\infty}^{\infty}\left(\frac{1}{2} u^{2}+\frac{1}{2} u v^{2}-\frac{1}{2} \sigma v_{x}^{2}\right)
$$

is the Hamiltonian.

We now construct the recursion operator for symmetries from the vector field $Z_{1}$. First of all

$$
\tilde{Z}_{1}=\Omega Z_{1}=\frac{1}{4}\left(\begin{array}{l}
t\left(4 \sigma v_{2}+v^{3}+6 u v\right)+x\left(2 u+v^{2}\right)+2 D^{-1}(u) \\
t\left(4 \sigma u_{2}+6 \sigma v v_{2}+3 v_{1}^{2}+3 u v^{2}+3 u^{2}\right)+x\left(2 \sigma v_{2}+2 u v\right) \\
+D^{-1}\left(4 \sigma v_{2}+2 u v\right)
\end{array}\right)
$$

and calculate $\tilde{Z}_{1}^{\prime}$ and $\left(\tilde{Z}_{1}^{\prime}\right)^{*}$, i.e., the Frechet derivative of $\tilde{Z}_{1}$ and its adjoint, from which we obtain

$$
\begin{aligned}
& T=\Omega^{-1}\left(\tilde{Z}_{1}^{\prime}-\tilde{Z}_{1}^{\prime *}\right) \\
& T=\left(\begin{array}{cc}
\frac{1}{2} v & \sigma D^{2}+u+\frac{1}{2} u_{1} D^{-1} \\
1 & \frac{1}{2} v+\frac{1}{2} v_{1} D^{-1}
\end{array}\right)
\end{aligned}
$$

i.e., the recursion operator for symmetries, which is in complete agreement with the result obtained by Itô.

\section{Nonlocal Symmetries and the Linearization of the Federbush Model}

As another application of the developed software, we construct nonlocal symmetries of the Federbush model. By mutilation of two interesting nonlocal symmetries, we obtain a transformation linearizing the Federbush model.

The Federbush model is described by

$$
\left(\begin{array}{cc}
i\left(\partial_{t}+\partial_{x}\right) & -m(k) \\
-m(k) & i\left(\partial_{t}-\partial_{x}\right)
\end{array}\right)\left(\begin{array}{l}
\psi_{k, 1} \\
\psi_{k, 2}
\end{array}\right)=k \lambda\left(\begin{array}{c}
+\left|\psi_{-k, 2}\right|^{2} \psi_{k, 1} \\
-\left|\psi_{-k, 1}\right|^{2} \psi_{k, 2}
\end{array}\right)_{(k= \pm 1)},
$$


where $\psi_{k}(x, t)(k= \pm 1)$ are two-component functions defined on $\mathbb{C}$. Introducing $U_{i}, V_{i}(i=1, \ldots, 4)$ by

$$
\begin{array}{ll}
\psi_{1,1}=U_{1}+i V_{1}, & \psi_{-1,1}=U_{3}+i V_{3}, \\
\psi_{1,2}=U_{2}+i V_{2}, & \psi_{-1,2}=U_{4}+i V_{4}
\end{array}
$$

system (5.1) is rewritten as a system of eight nonlinear partial differential equations

$$
\begin{aligned}
& U_{1 t}+U_{1 x}-m_{1} V_{2}=\lambda R_{4} V_{1}, \\
& V_{1 t}+V_{1 x}+m_{1} U_{2}=-\lambda R_{4} U_{1}, \\
& U_{2 t}-U_{2 x}-m_{1} V_{1}=-\lambda R_{3} V_{2}, \\
& V_{2 t}-V_{2 x}+m_{1} U_{1}=\lambda R_{3} U_{2}, \\
& U_{3 t}+U_{3 x}-m_{2} V_{4}=-\lambda R_{2} V_{3}, \\
& V_{3 t}+V_{3 x}+m_{2} U_{4}=\lambda R_{2} U_{3}, \\
& U_{4 t}-U_{4 x}-m_{2} V_{3}=\lambda R_{1} V_{4}, \\
& V_{4 t}-V_{4 x}+m_{2} U_{3}=-\lambda R_{1} U_{4},
\end{aligned}
$$

whereas, in (5.3),

$$
\begin{array}{ll}
m(1)=m_{1} ; & m(-1)=m_{2} \\
R_{i}=U_{i}^{2}+V_{i}^{2} & (i=1, \ldots, 4) .
\end{array}
$$

In [15], we obtained two infinitesimal symmetries

$$
\begin{aligned}
& V_{1}=-V_{1} \partial_{U_{1}}+U_{1} \partial_{V_{1}}-V_{2} \partial_{U_{2}}+U_{2} \partial_{V_{2}} \\
& V_{2}=-V_{3} \partial_{U_{3}}+U_{3} \partial_{V_{3}}-V_{4} \partial_{U_{4}}+U_{4} \partial_{V_{4}}
\end{aligned}
$$

giving rise to conserved currents [5]

$$
\begin{aligned}
& \left(R_{1}+R_{2}\right) \mathrm{d} x+\left(-R_{1}+R_{2}\right) \mathrm{d} t \\
& \left(R_{3}+R_{4}\right) \mathrm{d} x+\left(-R_{3}+R_{4}\right) \mathrm{d} t .
\end{aligned}
$$

We now formally introduce the nonlocal variables $p_{1}$ and $p_{2}$ by

$$
p_{1}=\int_{-\infty}^{x}\left(R_{1}+R_{2}\right) \mathrm{d} x, \quad p_{2}=\int_{-\infty}^{x}\left(R_{3}+R_{4}\right) \mathrm{d} x
$$

and construct the exterior differential system $I$, defined on

$$
\mathbb{R}^{20}=\left\{\left(x, t, U_{1}, \ldots, V_{4}, p_{1}, p_{2}, U_{1 x}, \ldots, V_{4 x}\right)\right\}
$$

and generated by the differential 1-forms

$$
\alpha_{1}=\mathrm{d} U_{1}-U_{1 x} \mathrm{~d} x-U_{1 t} \mathrm{~d} t,
$$




$$
\begin{aligned}
& \alpha_{2}=\mathrm{d} V_{1}-V_{1 x} \mathrm{~d} x-V_{1 t} \mathrm{~d} t, \\
& \alpha_{3}=\mathrm{d} U_{2}-U_{2 x} \mathrm{~d} x-U_{2 t} \mathrm{~d} t, \\
& \alpha_{4}=\mathrm{d} V_{2}-V_{2 x} \mathrm{~d} x-V_{2 t} \mathrm{~d} t, \\
& \alpha_{5}=\mathrm{d} U_{3}-U_{3 x} \mathrm{~d} x-U_{3 t} \mathrm{~d} t, \\
& \alpha_{6}=\mathrm{d} V_{3}-V_{3 x} \mathrm{~d} x-V_{3 t} \mathrm{~d} t, \\
& \alpha_{7}=\mathrm{d} U_{4}-U_{4 x} \mathrm{~d} x-U_{4 t} \mathrm{~d} t, \\
& \alpha_{8}=\mathrm{d} V_{4}-V_{4 x} \mathrm{~d} x-V_{4 t} \mathrm{~d} t, \\
& \alpha_{9}=\mathrm{d} p_{1}-\left(R_{1}+R_{2}\right) \mathrm{d} x-\left(-R_{1}+R_{2}\right) \mathrm{d} t, \\
& \alpha_{10}=\mathrm{d} p_{2}-\left(R_{3}+R_{4}\right) \mathrm{d} x-\left(-R_{3}+R_{4}\right) \mathrm{d} t,
\end{aligned}
$$

and the exterior derivatives $\mathrm{d} \alpha_{1}, \ldots, \mathrm{d} \alpha_{8} . \mathrm{d} \alpha_{9}, \mathrm{~d} \alpha_{10}$ are in $I$ by (5.6). The symmetry condition

$$
L_{V} I \subset I
$$

leads to an overdetermined system of partial differential equations for the components of the vector field $V$.

The Lie algebra of infinitesimal symmetries is generated by the vector fields

$$
\begin{aligned}
V_{1}= & \partial_{x}, \quad V_{2}=\partial_{t}, \\
V_{3}= & V_{1} \partial_{U_{1}}-U_{1} \partial_{V_{1}}+V_{2} \partial_{U_{2}}-U_{2} \partial_{V_{2}}, \\
V_{4}= & V_{3} \partial_{U_{3}}-U_{3} \partial_{V_{3}}+V_{4} \partial_{U_{4}}-U_{4} \partial_{V_{4}}, \\
V_{5}= & t \partial_{x}+x \partial_{t}+\frac{1}{2}\left(U_{1} \partial_{U_{1}}+V_{1} \partial_{V_{1}}-U_{2} \partial_{U_{2}}-V_{2} \partial_{V_{2}}\right)+ \\
& +\frac{1}{2}\left(U_{3} \partial_{U_{3}}+V_{3} \partial_{V_{3}}-U_{4} \partial_{U_{4}}-V_{4} \partial_{V_{4}}\right) \\
V_{6}= & \partial_{p_{1}}, \quad V_{7}=\partial_{p_{2}}, \\
V_{8}= & U_{1} \partial_{U_{1}}+V_{1} \partial_{V_{1}}+U_{2} \partial_{U_{2}}+V_{2} \partial_{V_{2}}+ \\
& +\lambda p_{1}\left(-V_{3} \partial_{U_{3}}+U_{3} \partial_{V_{3}}-V_{4} \partial_{V_{4}}+U_{4} \partial_{U_{4}}\right)+2 p_{1} \partial_{p_{1}}, \\
V_{9}= & \lambda p_{2}\left(V_{1} \partial_{U_{1}}-U_{1} \partial_{V_{1}}+V_{2} \partial_{U_{2}}-U_{2} \partial_{V_{2}}\right)+ \\
& +U_{3} \partial_{U_{3}}+V_{3} \partial_{V_{3}}+U_{4} \partial_{U_{4}}+V_{4} \partial_{V_{4}}+2 p_{2} \partial_{p_{2}} .
\end{aligned}
$$

The symmetries $V_{1}, \ldots, V_{5}$ are the classical local ones (cf. [3], (2.6)). We now compute the local 1-parameter group of symmetries associated to the vector field $V_{8}+V_{9}$. This leads to the following result (5.2)

$$
\begin{aligned}
& \tilde{U}_{1}(s)=\mathrm{e}^{s}\left(U_{1} \cos r_{1}+V_{1} \sin r_{1}\right), \\
& \tilde{V}_{1}(s)=\mathrm{e}^{s}\left(-U_{1} \sin r_{1}+V_{1} \cos r_{1}\right), \\
& \tilde{U}_{2}(s)=\mathrm{e}^{s}\left(U_{2} \cos r_{1}+V_{2} \sin r_{1}\right), \\
& \tilde{V}_{2}(s)=\mathrm{e}^{s}\left(-U_{2} \sin r_{1}+V_{2} \cos r_{1}\right),
\end{aligned}
$$




$$
\begin{aligned}
& \tilde{U}_{3}(s)=\mathrm{e}^{s}\left(U_{3} \cos r_{2}-V_{3} \sin r_{2}\right), \\
& \tilde{V}_{3}(s)=\mathrm{e}^{s}\left(U_{3} \sin r_{2}+V_{3} \cos r_{2}\right), \\
& \tilde{U}_{4}(s)=\mathrm{e}^{s}\left(U_{4} \cos r_{2}-V_{4} \sin r_{2}\right), \\
& \tilde{V}_{4}(s)=\mathrm{e}^{s}\left(U_{4} \sin r_{2}+V_{4} \cos r_{2}\right)
\end{aligned}
$$

or

$$
\begin{aligned}
& \tilde{\psi}_{1,1}=\mathrm{e}^{s} \psi_{1,1} \mathrm{e}^{i r_{1}} \\
& \tilde{\psi}_{1,2}=\mathrm{e}^{s} \psi_{1,2} \mathrm{e}^{i r_{1}} \\
& \tilde{\psi}_{-1,1}=\mathrm{e}^{s} \psi_{-1,1} \mathrm{e}^{-i r_{2}} \\
& \tilde{\psi}_{-1,2}=\mathrm{e}^{s} \psi_{-1,2} \mathrm{e}^{-i r_{2}} \\
& \tilde{p}_{1}(s)=\mathrm{e}^{2 s} p_{1}, \quad \tilde{p}_{2}(S)=\mathrm{e}^{2 s} p_{2},
\end{aligned}
$$

where in (5.11), $s$ is the group parameter and

$$
r_{1}=\frac{\lambda}{2} p_{2}\left(\mathrm{e}^{2 s}-1\right), \quad r_{2}=\frac{\lambda}{2} p_{1}\left(\mathrm{e}^{2 s}-1\right) ;
$$

$s=0$ representing the identity-map in (5.11).

By definition, the transformation (5.11) maps solutions of the Federbush model into solutions of the same system.

We now mutilate the transformations defined by (5.11) by deleting $\mathrm{e}^{s}$ in (5.11a) and by consequence of $(5.7) \mathrm{e}^{2 s}$ in $(5.11 \mathrm{~b})$ which results in the transformations

$$
\begin{aligned}
& \tilde{\psi}_{1,1}=\psi_{1,1} \mathrm{e}^{i r_{1}}, \quad \tilde{\psi}_{1,2}=\psi_{1,2} \mathrm{e}^{i r_{1}}, \\
& \tilde{\psi}_{-1,1}=\psi_{-1,1} \mathrm{e}^{-i r_{2}}, \quad \tilde{\psi}_{-1,2}=\psi_{-1,2} \mathrm{e}^{-i r_{2}}
\end{aligned}
$$

and its inverse.

An easy calculation now shows that if $\psi_{i, j}(i= \pm 1, j=1,2)$ is a solution of (5.1) then $\tilde{\psi}_{i, j}$ satisfies the following nonlinear system of partial differential equations

$$
\left(\begin{array}{cc}
i\left(\partial_{t}+\partial_{x}\right) & -m(k) \\
-m(k) & i\left(\partial_{t}+\partial_{x}\right)
\end{array}\right)\left(\begin{array}{c}
\tilde{\psi}_{k, 1} \\
\tilde{\psi}_{k, 2}
\end{array}\right)=s k \lambda\left(\begin{array}{cc}
+\left|\tilde{\psi}_{-k, 2}\right|^{2} & \tilde{\psi}_{k, 1} \\
-\left|\tilde{\psi}_{-k, 1}\right|^{2} & \tilde{\psi}_{k, 2}
\end{array}\right) \mathrm{e}^{2 s} .
$$

Now in case $s=0$ the nonlinear term in the right-hand side of (5.14) cancels and by consequence the transformation

$$
\begin{aligned}
& \tilde{\psi}_{1,1}=\psi_{1,1} \exp \left[\frac{-i \lambda}{2} p_{2}\right], \quad \tilde{\psi}_{1,2}=\psi_{1,2} \exp \left[\frac{-i \lambda}{2} p_{2}\right], \\
& \tilde{\psi}_{-1,1}=\psi_{-1,1} \exp \left[\frac{+i \lambda}{2} p_{1}\right], \quad \tilde{\psi}_{-1,2}=\psi_{-1,2} \exp \left[\frac{+i \lambda}{2} p_{1}\right],
\end{aligned}
$$

transform (5.1) into the linear system 


$$
\left(\begin{array}{cc}
\mathrm{i}\left(\partial_{\mathrm{t}}+\partial_{\mathrm{x}}\right) & -m(k) \\
-\mathrm{m}(\mathrm{k}) & i\left(\partial_{t}+\partial_{x}\right)
\end{array}\right)\left(\begin{array}{c}
\tilde{\psi}_{k, 1} \\
\tilde{\psi}_{k, 2}
\end{array}\right)=0 .
$$

\section{References}

1. Hearn, A. C.: REDUCE User's Manual (Version 3.0), The Rand Corporation, Santa Monica, 1983.

2. Frick, I. B.: Manual for Standard LISP on DEC-system 10 and 20, Symbolic Group of the University of Utah, 1978.

3. Kersten, P. H. M.: Infinitesimal symmetries: a computational approach, CWI Tract 34, Centre for Mathematics and Computer Science, Amsterdam, 1987.

4. Schwartz, F.: Automatically determining symmetries of partial differential equations, G. M. D. Institute F1, St Augustin, West Germany (preprint), 1984.

5. Shadwick, W. F.: The Bäcklund problem, symmetries and conservation laws for some nonlinear differential equations, PhD Thesis, King's College, London, 1979.

6. Vinogradov, A. M.: Local symmetries and conservation laws, Acta Appl. Math. 2 (1984), 21-78; Vinogradov, A. M. and Krasilshchik, I. S.: Nonlocal symmetries and the theory of coverings: an addendum to A. M. Vinogradov's 'Local symmetries and conservation laws', Acta Appl. Math. 2 (1984), 79-96.

7. Anderson, R. L. and Ibragimov, N. H.: Lie-Bäcklund Transformations in Applications, SIAM Studies in Applied Mathematics, Philadelphia, 1979.

8. Harrison, B. K. and Estabrook, F. B.: Geometric approach to invariance groups and solutions of partial differential systems, J. Math. Phys. 12 (1971), 653-666.

9. Kersten, P. H. M.: Infinitesimal symmetries and conserved currents for nonlinear Dirac equations, Memorandum 398, Twente University of Technology, The Netherlands, 1982, J. Math. Phys. 24 (1983), 2374-2376.

10. Kersten, P. H. M.: The computation of the infinitesimal symmetries for (extended) Vacuum Maxwell Equations, using Reduce 2, Memorandum 365, Twente University of Technology, The Netherlands, 1981;

Kersten, P. H. M. and Gragert, P. K. H.: Implementation of differential geometric objects and functions with an application to extended Maxwell equati ns, in J. Calmet (ed.), Proceedings Eurocam 1982, Lecture Notes in Computer Science, Vol. 144. Springer, Heidelberg, New York, Berlin, 1982.

11. Gragert, P. K. H., Kersten, P. H. M., and Martini, R.: Symbolic computations in applied differential geometry, Acta Appl. Math. 1 (1983), 43-77.

12. Harrison, B. K. and Estabrook, F. B.: Geometric approach to invariance groups and solutions of partial differential systems, J. Math. Phys. 12 (1971), 653-666.

13. Gragert, P. K. H.: Symbolic computations in prolongation theory, PhD Thesis, Twente University of Technology, Enschede, The Netherlands, 1981.

14. Itô, M.: Symmetries and Conservation Laws of the Classical Boussinesq equation, Phys. Lett. 104A (1984), 248-250.

15. Kersten, P. H. M.: Creating and annihilating Lie-Bäcklund transformations of the Federbush Model, J. Math. Phys. 27 (1987), 1139-1144.

16. Kersten, P. H. M.: Nonlocal symmetries and the linearization of the massless Thirring and the Federbush model, J. Math. Phys. 28 (1988), 1050-1053. 